\title{
Macroscopic X-ray powder diffraction imaging for the study of oil paintings
}

\author{
F. Vanmeert ${ }^{1,2}$, S. De Meyer ${ }^{1}$, A. Gestels ${ }^{1}$, S. Saverwyns ${ }^{2}$, K. Janssens ${ }^{1}$ \\ ${ }^{1}$ AXES Research Group, NANOLab Centre of Excellence, University of Antwerp, Belgium, ${ }^{2}$ Laboratory Department, Royal Institute \\ for Cultural Heritage, Brussels, Belgium
}

frederik.vanmeert@uantwerpen.be

A painting is made up of complex mixtures of materials, carefully selected by an artist, usually to create a specific optical illusion or esthetic effect. Depending on its material composition and the environmental conditions that a painting is subjected to, various chemical reactions can take place which cause the paint layers to deteriorate over time. Therefore, collecting reliable chemical information from a work of art is essential to understand its composition, past and ongoing conservation issues and to develop preservation strategies. In this sense, X-ray powder diffraction is an important tool as it allows for the direct identification of crystalline phases within the complex mixtures present in a painting [1]. However, an important limitation of this method has been the amount of material that needed to be sampled [2]. In the past decade a new trend has been set towards the application of elemental and chemical imaging techniques, such as macroscopic X-ray fluorescence (MA-XRF) and reflectance imaging spectroscopy (RIS), for the study of painted artefacts as they provide valuable information on the heterogeneous composition within complete paintings [3-5].

Following this trend, the AXES research group has developed a macroscopic X-ray powder diffraction (MAXRPD) imaging instrument that allows for the identification and visualization of the crystalline materials used in a painting in a non-invasive manner. This instrument uses a low power microfocus X-ray source ( $\mathrm{I} \mu \mathrm{S}$, Incoatec) combined with multilayer mirrors to obtain a slightly focused and fairly monochromatic X-ray beam in combination with a large area detector (PILATUS 200K, Dectris). By moving the painting and the instrument relative to each other, a large set of diffraction images (typically $>10000$ ) is collected following a rasterscanning approach. Subsequently, this large powder diffraction dataset is azimuthally integrated after which the resulting one dimensional $2 \theta$ spectrum at each data point is individually fitted with the XRDUA software package [6] using a model comprising all identified crystalline phases. By plotting the scaling factors as grey-scale values individual images that correspond to the distribution of the crystalline materials can be created [7].

The MA-XRPD instrument can be used in a transmission geometry, suitable for underlaying and strongly absorbing paint layers, or in reflection geometry, which is more sensitive for the (thin) pictorial layers. The latter also has the added advantage that larger works of art can be investigated as the painting remains stationary while the scanning head is translated in three dimensions. Typically a (short) dwell time of 10 seconds is used with a step size of 1-2 mm over a maximum scanning range of $30 \times 30 \mathrm{~cm}$.

The MA-XRPD instrument has been used within several museums on well-known masterpieces, such as Van Gogh's Sunflowers, Vermeer's Girl with a Pearl Earring, the Ghent altarpiece by the brothers Van Eyck and The Night Watch by Rembrandt. On these works, next to the visualization of the original pigments employed by the artists and later additions or overpaint, also various chemical alteration products that have formed within/on top of the paint layers could be identified. In some cases, the data collected with the MA-XRPD instrument can be exploited to yield other types of highly-specific information, such as the buildup of the paint layer or the orientation of the crystals on the paint surface. Furthermore, the collection of large datasets allows a reliable quantification of various pigment mixtures and to track their differences within and between artworks/time periods.

[1] Artioli, G. (2013). Rendiconti Lincei-Scienze Fisiche E Naturali, 24, S55.

[2] Madariaga, J. M. (2015). Anal. Methods, 7, 4848.

[3] Alfeld, M., \& Broekaert, J. A. C. (2013). Spectrochim. Acta, Part B, 88, 211.

[4] Alfeld, M., \& de Viguerie, L. (2017). Spectrochim. Acta, Part B, 136, 81.

[5] Trentelman, K. (2017). Annu. Rev. Anal. Chem., 10, 247.

[6] De Nolf, W., Vanmeert, F., \& Janssens, K. (2014). J. Appl. Crystallogr., 47, 1107.

[7] Vanmeert, F., De Nolf, W., De Meyer, S., Dik, J., \& Janssens, K. (2018). Anal Chem, 90, 6436.

Keywords: X-ray powder diffraction; imaging; quantification; chemical alteration; cultural heritage 